\title{
Avaliação dos Efeitos a Curto Prazo de Programas de Conscientização e de Terapia com Pravastatina em Indivíduos de Alto Risco para Doença Cardiovascular Provenientes de Consultórios Particulares
}

\author{
Raul D. Santos, Luiz O. do N ascimento, Raul C. Maranhão e Pesquisadores
}

São Paulo, SP

Objetivo - Avaliar a eficácia de programas de conscientização $(P C)$ sobre o controle de fatores de risco (FR) para doença cardiovascular (DCV).

Métodos - Pacientes hipercolesterolêmicos de alto risco para DCV foram divididos em 2 grupos durante 16 semanas. O grupo A ( $n=417,54,3 \pm 10,0$ anos, $55 \%$ homens) recebeu orientação verbal e escrita sobre controle de $F R$, e o grupo $B(n=180,54,4 \pm 10,9$ anos, $45 \%$ homens $)$ apenas orientação verbal. Todos os participantes receberam $10 \mathrm{mg} /$ dia de pravastatina por 12 semanas. Avaliaram-seo peso, pressão arterial, o colesterol total (CT) e frações, triglicérides, índices I e II de Castelli (CT/HDL-C e LDL-C/ $H D L-C)$ e escores de Framingham.

Resultados - No basal, A diferiu de B no HDL-C $(40,0 \pm 11,0 \mathrm{vs} 43,0 \pm 11,0 \mathrm{mg} / \mathrm{dl}, \mathrm{p}=0,013)$ e no índice I $(8,2 \pm 3,0$ vs $7,6 \pm 2,3, p=0,008)$. Após 16 semanas as variações \% foram maiores em A do que B no CT (-28,0 vs -25,0, $p<0,05), L D L-C$ (-29,0 vs -27,6, $p<0,05), H D L-C(+13,7$ $v s .+10,8, p<0,05)$ e indice I $(-39,0$ vs $-33,0 ; p<0,05)$. A pravastatina potencializou os efeitos da dieta sobre os lipides.

Conclusão - O PC parece ser mais eficaz a curto prazo, em reduzir os FR para DCV do que a orientação apenas verbal.

Palavras-chave: hipercolesterolemia, doença cardiovascular, fatores de risco, pravastatina

\section{The Evaluation of Short Term Effects of Awareness Programs and Pravastatin Therapy on Subjects from Private Clinics at High Risk for Cardiovascular Disease}

Purpose - To evaluate short-term efficacy of awareness programs $(A P)$ in reducing coronary heart disease riskfactors (CHDRF).

Methods - High risk hypercholesterolemic patients were divided in 2 groups during 16 weeks. Group A ( $n=417$, $54.3 \pm 10.0$ years, $55 \%$ males) received verbal and written orientation on CHDRF control, and group B $(n=180$, $54.4 \pm 10.9$ years, $45 \%$ males) received only verbal orientation. All participants received pravastatin $10 \mathrm{mg}$ q.d. for 12 weeks. The evolution of body weight, arterial pressure, lipid profile, Castelli's I and II indexes (TC/HDL and LDL/HDL), and Framingham scores were evaluated.

Results - At baseline, A had a lower HDL-C $(40.0 \pm 11.0 \mathrm{vs} 43.0 \pm 11.0 \mathrm{mg} / \mathrm{dl}, p=0.013)$ and a higher in $\operatorname{dex} I(8.2 \pm 3.0$ vs $7.6 \pm 2.3, p=0.008)$ than B. After 16 weeks, $A$ had greater change than $B$ in TC $(-28.0 \mathrm{vs}$ 25.0, $p<0.05), L D L-C$ (-29.0vs -27.6, $p<0.05), H D L-C$ levels $(+13.7 v s+10.8, p<0.05)$ and in the Castelli's Index (-39.0 vs -33.0; $p<0.05)$. In both groups pravastatin use potentialized the effects of diet on the lipid profile.

Conclusion - The AP seemed to be more effective than verbal orientation alone in CHDRF reduction at short-term.

Key-words: hypercholesterolemia, cardiovascular disease, risk factors, pravastatin

Arq Bras Cardiol, volume 69 (no 4), 225-230, 1997

Instituto do Coração do Hospital das Clínicas - FMUSP

Correspondência: Raul D. Santos - Incor - Laboratório de Pesquisa - Av. Dr. Enéas

C. Aguiar, 44 - 1ำ - 05403-000 - São Paulo, SP

Recebido para publicação em 13/6/97

Aceito em 6/8/97
A redução da prevalência da doença cardiovascular (DCV) nas últimas décadas deveu-se em parte a programas de prevenção ${ }^{1}$. A eficácia desses programas é maior quanto maior for o risco de desenvolver aterosclerose apresentado pelo indivíduo ${ }^{2-3}$. Apesar da tendência decrescente, a 
morbi-mortalidade por DCV permanece ainda muito elevada nos países ocidentais, incluindo o Brasil $^{4}$, o que pode ser decorrente da dificuldade de se tratar indivíduos possuidores de fatores de risco mas assintomáticos. Nesses casos, a motivação do paciente para efetuar as medidas preventivas é naturalmente pequena. A conscientização sobre as implicações de ter o fator de risco e do que deve ser feito para diminuí-lo torna-se, assim, imperativa. O objetivo deste estudo aberto, não randomizado, realizado em consultório foi de comparar a curto prazo duas estratégias de orientação para controle de fatores de risco ministradas a hipercolesterolêmicos de alto risco para DCV. Uma delas foi a habitual, com orientação apenas verbal e a outra com a orientação reforçada por folhetos informativos (conscientização) para o controle desses fatores. Todos os participantes foram submetidos à terapia com o inibidor da HMGCo-A redutase pravastatina.

\section{Métodos}

Foram convidados para participar como investigadores neste estudo 334 cardiologistas das regiões Sudeste e Nordeste do Brasil e o estudo realizado em seus consultórios durante o ano de 1996. Foram avaliados pacientes de ambos os sexos, portadores de hipercolesterolemia, sendo os critérios de inclusão no estudo: 1) idade entre 18 e 75 anos; 2) no caso das mulheres, não poderiam ter potencial de gravidez, ou deveriam estar fazendo uso de métodos adequados de anticoncepção, devendo apresentar resultado negativo de teste para gravidez no soro ou na urina dois dias antes do tratamento; 3) hipercolesterolemia definida como colesterol total (CT) do plasma $>260 \mathrm{mg} / \mathrm{dl}$ na seleção e $>240 \mathrm{mg} / \mathrm{dl}$ após quatro semanas de dieta fase I da American Heart Association; 4) indivíduos com presença de pelo menos três outros fatores de risco para DCV, além da hipercolesterolemia, como: a) hipertensão arterial sistêmica (HAS); b) diabetes mellitus tipo I ou II; c) sexo masculino $>45$ anos; d) tabagismo; e) inatividade física; f) obesidade (índice de massa corpórea $>30 \mathrm{~kg} / \mathrm{m}^{2}$ ); g) antecedente familiar de DCV; h) hipertrofia ventricular esquerda. Os critérios de exclusão foram: 1) indivíduos analfabetos ou de baixo nível socioeconômico nos quais o tratamento proposto pudesse ser prejudicado; 2) mulheres grávidas, em amamentação ou com potencial de gravidez sem o uso de métodos adequados de contracepção; 3) dependência do álcool (duas ou mais doses por dia); 4) infarto do miocárdio ou acidente vascular cerebral nos últimos três meses; 5) presença de hepatopatias, colelitíase sintomática, nefropatias, pancreatite crônica, doenças da tireóide, diabetes mellitus descompensado (glicemia de jejum $>140 \mathrm{mg} / \mathrm{dl}$ ) ou outras doenças que, segundo o médico investigador ou a bula da pravastatina, pudessem colocar os indivíduos sob risco ou interferir no resultado do estudo; 6) uso concomitante de hormônios, sais biliares, imunossupressores ou drogas hipolipemiantes. Havendo uso prévio destas últimas, deveriam ser suspensas por 30 dias, sendo os indivíduos reavaliados ao término do período; 7) alergia ou eventos adversos sérios decorrentes do uso prévio de inibidores da HMGCo-A redutase.

Foram realizadas cinco consultas médicas com intervalo de quatro semanas (total 20 semanas). Consulta 0 (pré estudo) - os participantes foram pré-selecionados, e baseados em seu perfil lipídico prévio, foi orientada verbalmente dieta pobre em gorduras saturadas e em colesterol (dieta fase I); consulta 1 - constando de anamnese e exame físico completo com ênfase na medida de peso e pressão arterial (PA) com o indivíduo deitado e sentado. Foram analisados eletrocardiogramas disponíveis para detecção de sobrecarga ventricular esquerda, assim como determinado o perfil lipídico: CT, colesterol da LDL (LDL-C), colesterol da HDL (HDL-C), colesterol de VLDL(VLDL-C) e triglicerídes (TG) do plasma. Existindo dúvida em relação a distúrbios hepáticos ou neuromusculares determinaram-se as aminotransferases e a creatinoquinase (CPK). Foi calculado o escore de risco de desenvolvimento de doença coronária, baseandose na tabela derivada do estudo de Framingham ${ }^{5}$, onde se atribuem pontos à idade, HDL-C, CT, pressão arterial sistólica (PAS) e presença de tabagismo, hipertrofia ventricular esquerda ou diabetes mellitus. O escore é baseado na soma desses fatores e quanto maior ele for maior o risco de doença arterial coronária (DAC) (vide apêndice I). Foram calculados também os índices de risco de Castelli I e II (CT/HDL-C e LDL-C/HDL-C, respectivamente) ${ }^{6}$. Os indivíduos foram separados em dois grupos conforme escolha ao acaso do médico investigador. No grupo A os participantes foram incluídos no programa de conscientização que consistia, além da orientação verbal sobre dieta, fumo, exercício e hipertensão, de folheto explicativo sobre dieta para reduzir o colesterol. Na consulta seguinte esses pacientes receberam outros folhetos informativos sobre colesterol, estresse, exercício físico, fumo e um folheto geral sobre a saúde do coração. No grupo B (tratamento usual) os participantes tiveram apenas orientação verbal sobre os fatores de risco e como controlá-los; consulta 2 - foram repetidas as avaliações clínica e colhidas amostras para determinação do perfil lipídico. Caso persistissem com os critérios de inclusão, era administrada a dose de $10 \mathrm{mg}$ de pravastatina em tomada única à noite (pravacol ${ }^{\mathrm{R}}$ ) durante quatro semanas. Foi anotada qualquer mudança ocorrida no tratamento medicamentoso. No grupo A, foram distribuídos os folhetos descritos anteriormente; consulta 3 - foi repetida a avaliação clínica. Naqueles participantes que se queixassem de sintomas neuromusculares foi determinada a CPK. No grupo A houve ainda reforço verbal da orientação dada quanto à redução dos fatores de risco ainda presentes e, no grupo B, tratadas apenas as queixas usuais dos participantes. Se tolerados os $10 \mathrm{mg}$ de pravastatina foram mantidos por mais quatro semanas; consulta 4 - foi repetido o exame clínico e laboratorial das avaliações 1 e 2 .

A avaliação da eficácia dos programas de conscientização foi baseada na evolução do peso corpóreo, da PA sistêmica (sistólica e diastólica), do perfil lipídico, dos índices de Castelli, dos escores de Framingham e na avaliação subjetiva de cada investigador quanto a eficácia da terapêu- 


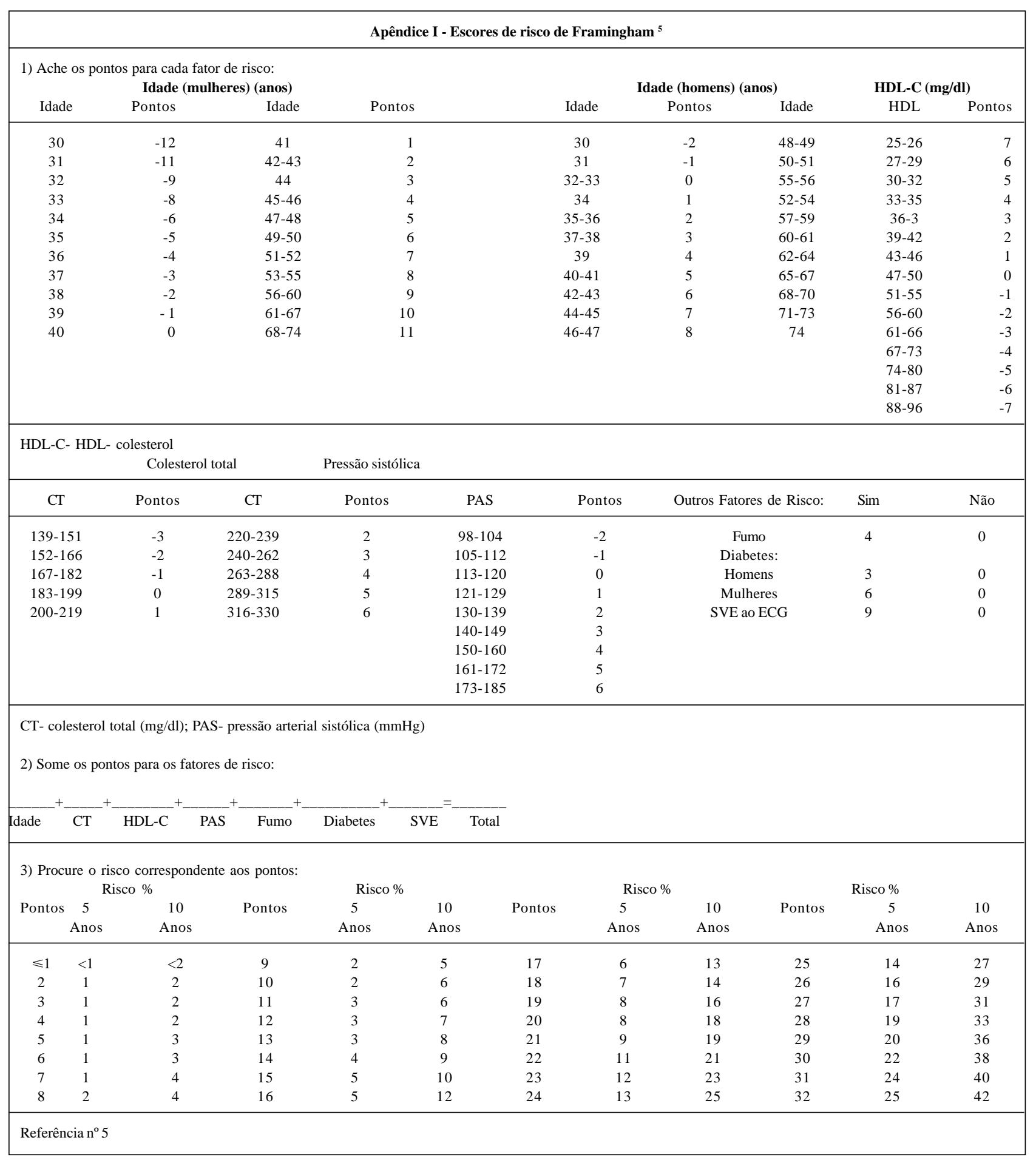

tica. Foi estimada também a capacidade dos regimes de tratamento em atingir a meta de LDL-C $<130 \mathrm{mg} / \mathrm{dl}$ nos participantes que não apresentassem DAC anteriormente (recomendação para a prevenção primária em indivíduos com >2 fatores de risco para DCV, segundo o consenso de dislipidemias da Sociedade Brasileira de Cardiologia) ${ }^{7}$.

$\mathrm{Na}$ avaliação dos eventos adversos do tratamento foram considerados eventos adversos, doenças, sintomas ou sinais que aparecessem ou piorassem no decorrer do estu- do. Esta consideração foi feita, independentemente, dos investigadores acreditarem ou não no relacionamento com a droga do estudo. Foram consideradas três categorias de intensidade de eventos: leve (sintomas ou sinais toleráveis), moderada (desconforto suficiente para interferir nas atividades habituais) e grave (incapacitante com inabilidade para desempenhar as atividades habituais). A avaliação dos eventos adversos foi feita por sinais e sintomas clínicos, pelo seguimento das enzimas musculares quando necessá- 
rio e pela necessidade de suspensão da pravastatina durante o estudo.

A determinação do perfil lipídico no plasma foi realizada em laboratórios de confiança dos investigadores após jejum de $12 \mathrm{~h}$. OCT, o HDL-C e os TG foram determinados por métodos enzimáticos. O LDL-colesterol foi calculado, utilizando-se a fórmula de Friedewald para níveis de triglicérides (TG) de até $400 \mathrm{mg} / \mathrm{dl}^{8}$. Caso necessário foram determinados também os níveis de aminotransferases e CPK através de métodos cinéticos. Os médicos foram orientados para encaminhar os indivíduos aos mesmos laboratórios durante todo o estudo (cinco consultas).

Quanto a análise estatística, nas tabelas os dados são apresentados como média \pm desvio padrão da média. As variações dos parâmetros analisados se tratam das variações das médias em percentual $(\Delta \%)$. As variáveis qualitativas foram analisadas pelo teste do chi-quadrado e as variáveis contínuas pelos testes t de Student e análise de medidas repetitivas. Foi possível então separar os efeitos dos tratamentos no tempo e nos grupos. A significância foi aceita a nível de $5 \%$.

Foi obtido consentimento informado para participação neste estudo em $98 \%$ dos indivíduos.

\section{Resultados}

Foram avaliados 597 indivíduos divididos em 417 no grupo A e 180 no grupo B. Não houve diferença em relação a idade e raça dos participantes porém, havia mais homens em A do que em B ( $55 \%$ vs $42 \%, p=0,0033)$. Os grupos eram similares em relação ao grau de instrução, percentual de obesos, número de fumantes, sedentários, diabéticos, hipertensos, portadores de hipertrofia ventricular esquerda

\begin{tabular}{|c|c|c|c|c|}
\hline \multicolumn{5}{|c|}{ Tabela I - Características da população } \\
\hline & & Grupo A & Grupo B & $\mathrm{p}$ \\
\hline \multicolumn{2}{|l|}{ Idade } & $54,3 \pm 10,0$ & $54,4 \pm 10,9$ & NS \\
\hline \multirow[t]{2}{*}{ Sexo } & Feminino $(\%)$ & 45,0 & 58,0 & 0,0033 \\
\hline & Masculino (\%) & 55,0 & 42,0 & \\
\hline \multicolumn{5}{|l|}{ Raça } \\
\hline & Branca $(\%)$ & 85,0 & 83,0 & \\
\hline & Não Branca (\%) & 12,1 & 15,5 & NS \\
\hline & Asiática (\%) & 2,9 & 1,5 & \\
\hline \multicolumn{5}{|c|}{ Grau de Instrução } \\
\hline & $1 \%$ e $2^{\circ}$ Graus $(\%)$ & 67,8 & 68,0 & NS \\
\hline & Universitários (\%) & 32,2 & 32,0 & \\
\hline \multicolumn{2}{|c|}{$\mathrm{IMC}>30 \mathrm{~kg} / \mathrm{m}^{2}(\%)$} & 51,0 & 48,0 & NS \\
\hline \multicolumn{2}{|c|}{$\operatorname{HAS}(\%)$} & 65,0 & 61,0 & NS \\
\hline \multicolumn{2}{|c|}{$\mathrm{DM}(\%)$} & 20,0 & 17,0 & NS \\
\hline \multicolumn{2}{|c|}{ Fumo $(\%)$} & 54,0 & 52,0 & NS \\
\hline \multicolumn{2}{|c|}{ Inatividade $(\%)$} & 91,0 & 90,0 & NS \\
\hline \multicolumn{2}{|c|}{ HVE (\%) } & 24,6 & 31,0 & NS \\
\hline \multicolumn{2}{|c|}{ Antecedente familiar (\%) } & 56,5 & 51,0 & NS \\
\hline \multicolumn{4}{|c|}{ Tratamento medicamentoso } & NS \\
\hline
\end{tabular}

\begin{tabular}{|c|c|c|c|}
\hline \multicolumn{4}{|c|}{ Tabela II - Doenças prévias } \\
\hline & Grupo A & Grupo B & $\mathrm{p}$ \\
\hline Cardiovasculares & 44,5 & 43,55 & NS \\
\hline Dermatológicas & 2,73 & 3,23 & NS \\
\hline Gastrointestinais & 11,36 & 11,29 & NS \\
\hline Genitourinárias & 5,45 & 4,84 & NS \\
\hline Respiratórias & 5,45 & 9,27 & NS \\
\hline Vasculares Periféricas & 11,36 & 8,47 & NS \\
\hline Outras & 19,09 & 19,35 & NS \\
\hline
\end{tabular}

ou antecedentes familiares para DAC. A presença de outras doenças concomitantes também foi similar (tab. I e II). O uso de medicamentos anti-hipertensivos e ou hipolipemiantes previamente ao estudo era igual nos grupos (tab. I e III). No grupo A, 54 indivíduos (13,0\%) eram portadores de DAC e no grupo B, $8(13,6 \%)$, p=ns. Em relação ao perfil lipídico na consulta 1 , o grupo $A$ apresentava valores menores de HDL-C que o grupo B ( $\Delta \%=-6, \mathrm{p}=0,013)$. Este fato se refletiu em um maior índice I de Castelli nesses pacientes (tab. IV). Os índices de risco de Framingham eram 18,1 1 7,6e $18,3 \pm 8,8$, respectivamente em $\mathrm{A}$ e B $(\mathrm{p}=\mathrm{ns})$.

Quando comparamos os dados das consultas 1 e 2 (efeito isolado das medidas dietéticas e de estilo de vida), observamos que houve redução significativa e similar entre A e B (efeito no tempo) no peso, pressão arterial diastólica (PAD) ePAS, CT, LDL-Ce TG. OHDL-C aumentou de forma similar nos grupos (tab. III). Quando os pacientes foram avaliados, independentemente do grupo, verificamos que a introdução da pravastatina potencializou de forma importante o efeito das medidas dietéticas e de estilo de vida no perfil lipídico (consultas 2 vs 4) $\Delta \%$ : CT (-9,4 vs -19,0), LDLC (-9,0 vs -21,4), TG (-13,0 vs -15,5) e HDL-C (+3,5 vs +7,5) todos os valores com $\mathrm{p}<0,05$. Quando comparamos os grupos A e B entre as consultas 1 e 4 observamos que existiu diminuição significativa e similar (efeito no tempo) do peso, frequiência cardíaca, PAD e PAS além dos níveis de TG e VLDL-C (tab. IV e V). Nos indivíduos do grupo A, a terapêutica mostrou-se mais efetiva em reduzir o CT (-28,0\% vs $-25 \%, \mathrm{p}<0,05)$ e oLDL-C (-29,0\% vs $-27,6 \%$, p<0,05) naconsulta 4. OHDL-C aumentou mais em A do que em B (+13,7\% $\mathrm{vs}+10,8 \%, \mathrm{p}<0,05)$. O índice I de Castelli foi reduzido de forma mais intensa no grupo A do que em B (-39,0\% vs $-33,0 \%)$. Os valores dos lipídeos e dos índices de Castelli encontram-se na tabela IV. Quando avaliamos os indivíduos

\begin{tabular}{|lrcc|}
\hline \multicolumn{3}{|c|}{ Tabela III - Medicamentos prévios ao estudo } \\
\hline & Grupo A & Grupo B & $\mathrm{p}$ \\
\hline Diuréticos & 13,47 & 13,4 & $\mathrm{NS}$ \\
Betabloqueadores & 3,63 & 5,67 & $\mathrm{NS}$ \\
Inibidores da ECA & 22,28 & 24,74 & $\mathrm{NS}$ \\
Bloqueadores de cálcio & 15,54 & 13,92 & $\mathrm{NS}$ \\
Simpatolíticos & 2,07 & 3,61 & $\mathrm{NS}$ \\
Estatinas & 21,76 & 19,59 & $\mathrm{NS}$ \\
Fibratos & 15,54 & 16,49 & $\mathrm{NS}$ \\
Outros & 5,71 & 2,58 & $\mathrm{NS}$ \\
\hline
\end{tabular}




\begin{tabular}{|c|c|c|c|c|c|c|}
\hline \multicolumn{7}{|c|}{ Tabela IV - Evolução dos lípides e dos índices de risco } \\
\hline & & Consulta 1 & Consulta 2 & Consulta 4 & $\begin{array}{c}p \\
\text { Consulta } \\
4 \text { vs1 }\end{array}$ & $\begin{array}{c}\mathrm{p} \\
\text { A vs B } \\
\text { consulta } 4\end{array}$ \\
\hline \multirow[t]{2}{*}{ CT } & A & $307,0 \pm 55,0$ & $278,0 \pm 34,0$ & $219,0 \pm 28,0$ & $<0,001$ & 0,005 \\
\hline & B & $304,0 \pm 46,0$ & $276,0 \pm 39,0$ & $228,0 \pm 37,0$ & $<0,001$ & \\
\hline \multirow{2}{*}{ LDL-C } & A & $201,0 \pm 43,0$ & $184,0 \pm 37,0$ & $142,0 \pm 29,0$ & $<0,001$ & 0,035 \\
\hline & B & $204,0 \pm 43,0$ & $185,0 \pm 36,0$ & $148,0 \pm 31,0$ & $<0,001$ & \\
\hline \multirow{2}{*}{ HDL-C } & A & $40,0 \pm 11,0^{*}$ & $42,0 \pm 10,0^{* * *}$ & $46,0 \pm 9,0$ & $<0,001$ & NS \\
\hline & B & $43,0 \pm 11,0$ & $45,0 \pm 10,0$ & $47,0 \pm 11,0$ & $<0,001$ & \\
\hline \multirow[t]{2}{*}{ TG } & A & $244,0 \pm 144,0$ & $213,0 \pm 121,0$ & $177,0 \pm 97,0$ & $<0,001$ & NS \\
\hline & B & $232,0 \pm 116,0$ & $201,0 \pm 87,0$ & $173,0 \pm 75,0$ & $<0,001$ & \\
\hline \multirow[t]{2}{*}{ Castelli I } & A & $8,2 \pm 3,0^{\#}$ & $7,0 \pm 1,9^{\# \#}$ & $5,0 \pm 1,3$ & $<0,001$ & NS \\
\hline & B & $7,6 \pm 2,3$ & $6,5 \pm 1,7$ & $5,0 \pm 1,4$ & $<0,001$ & NS \\
\hline \multirow[t]{2}{*}{ Castelli II } & A & $5,3 \pm 1,8$ & $4,6 \pm 1,5^{\circ}$ & $3,2 \pm 1,0$ & $<0,001$ & NS \\
\hline & $\mathrm{B}$ & $5,1 \pm 1,8$ & $4,3 \pm 1,3$ & $3,3 \pm 1,1$ & $<0,001$ & \\
\hline \\
\hline & \multicolumn{6}{|c|}{ Framingham } \\
\hline & A & $18,1 \pm 7,6$ & $16,4 \pm 7,7$ & $11,6 \pm 7,7$ & $<0,001$ & NS \\
\hline & B & $18,3 \pm 8,8$ & $18,8 \pm 7,4$ & $12,9 \pm 8,9$ & $<0,001$ & \\
\hline
\end{tabular}

\begin{tabular}{|c|c|c|c|c|c|c|c|}
\hline \multicolumn{8}{|c|}{ Tabela V - Evolução do peso corpóreo e da pressão arterial } \\
\hline & & Consulta 1 & Consulta 2 & Consulta 3 & Consulta 4 & $\begin{array}{c}\mathrm{p} \\
\text { Consulta } \\
4 \text { vs } 1\end{array}$ & $\begin{array}{c}\mathrm{p} \\
\mathrm{A} \text { vs } \mathrm{B}\end{array}$ \\
\hline Peso & A & $77.4 \pm 13,6$ & $75,4 \pm 13,1$ & $74,4 \pm 12,5$ & $73,4 \pm 12,1$ & $<0,001$ & NS \\
\hline$(\mathrm{Kg})$ & B & $75,5 \pm 13,6$ & $73,8 \pm 12,9$ & $73,1 \pm 12,6$ & $72,1 \pm 12,6$ & $<0,001$ & \\
\hline PAS & A & $142,0 \pm 18,0$ & $135,0 \pm 13,0$ & $132,0 \pm 13,0$ & $130,0 \pm 12,0 * *$ & $<0,001$ & NS \\
\hline$(\mathrm{mmHg})$ & B & $142,0 \pm 20,0$ & $136,0 \pm 15,0$ & $134,0 \pm 15,0$ & $131,0 \pm 13,0$ & $<0,001$ & \\
\hline PAD & A & $89,0 \pm 11,0$ & $85,0 \pm 8,0$ & $83,0 \pm 7,0$ & $82,0 \pm 7,0$ & $<0,001$ & NS \\
\hline$(\mathrm{mmHg})$ & $\mathrm{B}$ & $89,0 \pm 11,0$ & $85,0 \pm 9,0$ & $84,0 \pm 7,0$ & $83,0 \pm 7,0$ & $<0,001$ & \\
\hline
\end{tabular}

sem antecedentes pessoais para DCV, verificamos que o tratamento reduziu de forma similar a percentagem de pacientes com LDL ${ }^{3} 130 \mathrm{mg} / \mathrm{dl}$ nos dois grupos (de $97 \%$ para $69 \% *$ em Ae de $98 \%$ para $74 \% *$ em B, $* \mathrm{p}<0,001$ vs consulta 1 e $p=n s$ A vs B). Da mesma maneira, os índices de Framingham foram reduzidos de forma similare significativa no tempo, não havendo efeito do grupo (tab. IV). Segundo os médicos investigadores, a eficácia do tratamento foi maior no grupo A do que no $B(92,6 \%$ vs $81,5 \%, p=0,0004)$.

Em ambos os grupos não houve diferença nos eventos adversos do tratamento, que foram considerados como leves, não sendo atribuídos à pravastatina. A pravastatina foi suspensa em $0,4 \%$ dos indivíduos no grupo A e em $0,5 \%$ no grupo $B(p=n s)$. Não houve alteração significativa na medicação concomitante dos participantes de ambos os grupos em relação ao basal (dados não mostrados).

\section{Discussão}

Excetuando-se o predomínio do sexo masculino no grupo A, os dois grupos tinham incidência de fatores de risco semelhantes, o que nos permitiu a comparação dos dois esquemas de tratamento, apesar de ser um estudo aberto não randomizado. A população estudada é de alto risco para DCV, já que além das elevados níveis de LDL-C tinha também alta prevalência de HAS, obesidade, sedentarismo e tabagismo. A grande maioria dos envolvidos neste estudo apresentava valores de LDL-C acima dos preconizados para a prevenção primária pelo Consenso de Dislipidemias da Sociedade Brasileira de Cardiologia ${ }^{7}$. Além do LDL-C, orisco de DAC é mostrado pelos valores dos índices de Castelli e, de uma maneira mais abrangente, pelos escores de risco deFramingham.

Quando comparamos os grupos A e B, o grupo A apresentava valores menores de HDL-C e, conseqüentemente, um maior índice I de Castelli que o grupo B. Possivelmente, a maior prevalência de homens em A deva ter influenciado neste achado. Estudos mostram que os valores de HDL-C são constantemente mais baixos na população masculina ${ }^{9,10}$ mesmo quando comparados aos das mulheres na pós-menopausa.

Nosso estudo demonstrou que mesmo em quatro me- 
ses é possível agir sobre os fatores de risco de uma população em nível de consultório. Nos dois grupos houve redução do peso, da PA e melhora do perfil lipídico. Os dados indicam que quanto maior foi o grau de informação do indivíduo sobre como reduzir os fatores de risco, melhor o efeito no perfil lipídico. A maior eficácia do tratamento no grupo $\mathrm{A}$, em comparação com o grupo $\mathrm{B}$, foi expressa por maiores reduções do colesterol, LDL-C e por um maior aumento do HDL-C. Estes se refletiram numa maior redução do índice I de Castelli que era maior em A e ao término do tratamento se tornou similar ao do grupo B. É provável que a maior disponibilidade de informações, principalmente quanto a dieta para hipercolesterolemia (folheto), tenha influenciado nos resultados. Neste sentido, os outros determinantes que poderiam ter influenciado a curto prazo os valores do colesterol plasmático, como a perda de peso e a dosagem da pravastatina, foram similares nos grupos. Isto deixa a orientação dietética como a única variável identificável na resposta obtida. Vale salientar que a orientação dietética seguida dava mais ênfase à substituição do que para a subtração de alimentos, o que explicaria em parte, a queda similar do peso nos dois grupos. Embora as diferenças atingidas entre os grupos sejam pequenas (cerca de 3\% no CT), devemos lembrar que em média cada $1 \%$ de queda do CT plasmático equivale a $2-3 \%$ de redução do risco de eventos coronários ${ }^{11}$. Conseqüentemente, a diminuição do risco de DAC no grupo A seria de 6 a $9 \%$ maior do que no grupo B.

Em nosso estudo, 10mg de pravastatina associados a dieta causaram a redução de cerca de $26,5 \%$ no CT e de $28,5 \%$ no LDL-C. Os resultados foram obtidos sem efeitos colaterais importantes. A redução do colesterol atingida em nosso estudo foi similar a outras séries que avaliaram os efeitos da pravastatina no nosso meio ${ }^{12,13}$. Recentemente, foram divulgados os resultados do estudo de prevenção pri- mária do Oeste da Escócia ${ }^{14}$ que avaliou por cinco anos, 6595 homens de meia idade portadores de hipercolesterolemia. O tratamento com a pravastatina reduziu em média $20 \%$ oCT e $26 \%$ o LDL-C. A melhora do perfil lipídico acompanhou-se de redução de: $31 \%$ nos eventos coronários (infarto do miocárdio, angina, angiografia, angioplastia e revascularização cirúrgica), $33 \%$ na mortalidade coronária e $22 \%$ na mortalidade global. As curvas de mortalidade entre o grupo tratado e o grupo placebo começaram a se separar já com seis meses de tratamento nesse estudo. Assim, acreditamos que se mantidos os valores atingidos em nosso estudo, no decorrer do tempo, haverá benefício para os pacientes aqui avaliados. Entretanto, como a nossa população apresentava valores muito elevados da colesterolemia na consulta 1 , em 70,5\% dos participantes, não se chegou a atingir os níveis de LDL-C preconizados pelo consenso após quatro meses de tratamento, não sendo, portanto, o tratamento totalmente eficaz. Para atingirmos os níveis preconizados, seria necessário aumentar a dosagem de pravastatina ou associá-la a um outro medicamento redutor de colesterol.

Para finalizar, devemos levar em consideração as limitações de nosso estudo, que incluem variações no perfil lipídico decorrentes da falta de um laboratório central para as determinações dos lípides e uma possível escolha de pacientes mais susceptíveis ou não ao tratamento em algum dos grupos, concluindo que, quanto mais informações o paciente tiver sobre o seu risco de doença mais motivado ele ficará para reduzi-lo.

\section{Agradecimentos}

Aos Laboratórios Bristol Myers Squibb do Brasil e às estaticistas Paula G. Strassman e Sandra R. Malagutti pela análise estatística.

\section{Referências}

1. American Heart Association: Heart and Stroke Facts, 1995. Statistical Supplement. Dallas, American Heart Association, 1994.

2. Siegel D, Grady D, Browner WS, Hulley SB - Risk factor modification after myocardial infarction. Ann Intern Med 1988; 109: 213-18.

3. Rossouw JE, Lewis B, Rifkind BM-The value of lowering cholesterol after myocardial infarction. N Eng J Med 1990; 323: 1112-19.

4. Lotufo PA - Epidemiologia das doenças cardíacas no Brasil: histórico, situação atual e proposta de modelo teórico. Rev Soc Cardiol Est SP 1996;5:541-7.

5. Anderson KM, Wilson PWF, Odell PM, Kannel WB - An updated coronary risk profile. Circulation 1991; 83: 356-62.

6. Castelli WP, Garrison RJ, Wilson PWF, Abbot RD, Kalousdian S, Kannel WN Incidence of coronary heart disease and lipoprotein cholesterol levels: the Framingham Heart Study. JAMA 1986; 256: 2835-8.

7. 2ํㅡㄹ Consenso Brasileiro de Dislipidemias. Arq Bras Cardiol 1996; 67: 109-28.

8. Friedwald WT, Levy RI, Fredrickison DS - Estimation of the concentration of low density lipoprotein cholesterol in plasma, without the use of preparative ultracentrifuge. Clin Chem 1972; 18: 499-52.
9. Wilson PWF, Christiansen JC, Anderson KM et al - Impact of National Guidelines for Cholesterol Risk Factor Screening. The Framingahm Offspring Study. JAMA 1989; 262: 41-4.

10. Kannel WB - Range of serum cholesterol values in the population developing coronary artery disease. Am J Cardiol 1995: 75: 69C-77C.

11. Expert Panel - Report of the National Cholesterol Education Program Expert Panel on detection, evaluation, and treatment of high blood cholesterol in adults. Arch Intern Med 1988; 148: 36-69.

12. Barretto ACP, Wajngarten M, Gebara OCE et al - Fatores de risco em pacientes idosos selecionados por médicos da comunidade para tratamento hipolipemiante. Arq Bras Cardiol 1996; 67: 93-8.

13. Barretto ACP - Ensaio clínico multicêntrico da pravastatina em pacientes com hipercolesterolemia e múltiplos fatores de risco. Arq Bras Cardiol 1997; 68: 6571.

14. Shepherd J, Cobbe S M, Ford I et al - Prevention of coronary heart disease with pravastatin in men with hypercholesterolemia. N Eng J Med 1995; 333 : 1301-7. 